\title{
STABILITY OF A COLLISIONLESS STELLAR DISK
}

\author{
E. GRIV
}

Department of Physics, Ben-Gurion University, Israel

The study of stability of the stellar disks of flat galaxies is the first step towards an understanding of the phenomena of spiral structure. To explain the spiral pattern, Lin and Shu (1964) further developed the Lindblad's idea of density waves by considering the spiral structure as the collective effect in a self-gravitating system. As an extension to the original Lin-Shu theory, unstable spiral modes have been obtained from an asymptotic analysis (e.g., Bertin [1980]). In such "modal theory", the Jeans-type instability (even weak) is important to the maintenance of spiral structure.

However, the hypothesis that density waves are excited by the ordinary Jeans instability in a collisionless disk of stars encounters a severe challenge. By carrying out a nonlinear stability analysis, Griv et al. (1994) have proved that under the influence of aperiodically growing waves of a Jeans nature, the dispersion of residual (random) velocities of stars increases. Because the Jeans instability is characterized by the critical value of dispersion, this rise in turn leads to a decrease in the growth rate of the wave amplitude and, as a result, the gravitational instability will be switched off. Thus, the Jeans-unstable spiral waves in a stellar disk have to be short-lived.

From the observational point of view, the suggestion that many spiral structures in galaxies do not appear to be organized overall grand designs has become popular recently. Accordingly, the most of spiral galaxies may be divided into two types: regular spirals and ragged, flocculent ones. As for me, the existence of regular and flocculent galaxies reflects a different nature for the spiral arms in those systems.

Below, I describe the results of the theoretical analysis which has been done mainly to understand the structure in the stellar disk of flocculent galaxies (Griv and Peter 1994, Astrophys. J., submitted). We shown that in the nonlocal asymptotic approximation the generalized dispersion relation may be written as follows 


$$
\epsilon^{2} \frac{d^{2} \delta \Phi}{d r^{2}}+Q(\omega, k, r) \delta \Phi=0,
$$

where $\delta \Phi$ is the amplitude of the gravity perturbation, $\omega$ is the complex eigenfrequencies of excited waves, $k$ is the real wavenumber, $r$ is the radial coordinate, and $\epsilon^{2} \ll 1$. In analogy to plasma physics, the complex eigenvalues $\omega$ of the discrete modes that are spatially bounded are determined from (1) by means of the quasi-classical quantization rules of Bohr-Sommerfeld. The existence of discrete modes can be demonstrated by comparing (1) with the one-dimensional stationary Schrödinger equation. Assuming that $Q(\omega, k, r)$ is an almost real function, one can write relations defining the frequency and the growth rate of oscillations

$$
\int_{r_{2}}^{r_{1}} \operatorname{Re}\{\sqrt{Q}\} d r=\pi(n+1 / 2)
$$

and

$$
\operatorname{Im}\{\omega\}=-\frac{\int_{r_{2}}^{r_{1}} \operatorname{Im}\{\sqrt{Q}\} d r}{\int_{r_{2}}^{r_{1}} \frac{\partial}{\partial \omega} \operatorname{Re}\{\sqrt{Q}\} d r},
$$

and $n$ is a positive number. Here the domain of integration is the range of "transparency" between the turning points $r_{1}$ and $r_{2}$ which are defined by $Q\left(r_{1}\right)=Q\left(r_{2}\right)=0$. Equation (3) should be used only when the frequency, defined by Eq. (2), is real. Imaginary part of $\omega$, that is, $\operatorname{Im}\{\omega\}$, which defines the Landau growth of oscillations, is equal to zero when the drift motion is neglected. In turn, that minor drifting motion, in additional to the regular circular motion, is analogous to the magnetic drift of charged particles of a plasma in an inhomogeneous magnetic field, and is related to the differential rotation of the disk (Grivnev 1988).

As it follows from (2) and (3), stellar disks of flat galaxies besides experiencing the usual gravitational Jeans instability (when the frequency from (2) is imaginary), can experience a specific kinetic instability of the Landau type. The etiology of this instability is the resonant interaction of drifting stars with the field of the Jeans-stable and gradient waves. The kinetic instability can be considered to be a generating mechanism for density waves, thereby leading to spiral-like patterns and dynamical relaxation in the stellar disk of disk-shaped galaxies.

\section{References}

Bertin, G. (1980) Phys. Rep., 61, p. 1

Griv, E., Chiueh, Tz. and Peter, W. (1994) Physica A, 205, p. 299

Grivnev, E.M. (1988) Soviet Astron., 32, p. 139

Lin, C.C. and Shu, F.H. (1964) Astrophys. J., 140, p. 646 